\title{
A New Spectrum Management Scheme for Road Safety in Smart Cities
}

\author{
Ayoub Alsarhan, Ahmed Al-Dubai Senior Member, IEEE, Geyong Min, Albert Y. Zomaya Fellow, \\ IEEE, and Mohammad Bsoul
}

\begin{abstract}
Traffic management in roads is one of the major challenges faced in developing efficient intelligent transportation systems. Recently, wireless networks have received significant attention for tackling this challenge. However, wireless technologies face the well-known spectrum scarcity problem due to the explosive demand for radio resources. To overcome this challenge, this study presents a novel intelligent traffic control system, utilising the unused spectrum. Unlike existing works, the spectrum owners in this study hire free spectrum to drivers. The hired spectrum is deployed to build a short-range cost-effective wireless communication for monitoring traffic and enabling drivers to exchange warning messages, and thus enhancing road safety. Our objectives include minimizing crash probability, utilizing unused spectrum, and enabling spectrum owners to generate extra revenue. Numerical analysis demonstrates the capability of our approach to minimize the crash probability among vehicles under different operating road conditions.
\end{abstract}

Index Terms-Intelligent transportation systems, Dynamic spectrum access, Road traffic control, Crash probability, Wireless mesh networks.

\section{INTRODUCTION}

$\mathrm{N}_{\mathrm{t}}$ OWADYS, there is a tremendous growth of motorization over the world and traffic congestion which has been growing significantly on public roads. Smart city initiatives encourage embedding information and communication technologies across all city functions to promote service quality and facilitate smart digital inclusions. One application of the smart city paradigm is developing intelligent transportation systems (ITS), in which the main concern is to create a safe and smart transportation system for drivers [1-4].

Currently, traffic management systems use fixed camera video to monitor traffic flows. However, these systems are power hungry and require high bandwidth for video streams [1-4]. In addition, intrusive cameras require high installation and incur high maintenance costs. Furthermore, weather conditions degrade the performance of such systems considerably. Traditional traffic monitoring systems gain more computing power time, but are still inadequate to meet the requirements of ITS. Indeed, vehicles do not suffer from resource limitation constraints. Modern vehicles are equipped with small, low-cost devices that can sense the events taking place on the road and exchange data wirelessly [1-5]. In order to reduce traffic congestion, vehicles need to exchange road status that includes

- AyoubAlsarhan is with the Dept. Computer Information Systems, Hashemite University, Jordan, E-mail: ayoubm@hu.edu.jo.

- Ahmed Al-Dubai is with the School of Computing, Edinburgh Napier University, 10 Colinton Road, Edinburgh, EH10 5DT, U.K. E-mail: a.al-dubai@napier.ac.uk.

- Geyong Min is with Dept. Computer Science, University of Exeter, Exeter, EX4 4QF, U.K, Email: g.min@exeter.ac.uk

- Albert.Y. Zomaya is with School of Information Technologies, University of Sydney, E-mail: albert.zomaya@sydney.edu.au

- MohammadBsoulis with the Dept. Computer Information Systems, Hashemite University, Jordan, E-mail: mbsoul@hu.edu.jo. changes in traffic patterns and unforeseen obstacles such as accidents, road blocks, and traffic jams. Sensors enable drivers to share road status immediately. Real-time traffic information helps driver to adapt to the environment immediately [1-4]. Using traffic information, drivers can plan their routes, and driving speeds. However, adding these services will create a spectrum burden into the well-known scarcity problem in wireless technology [5-8]. To deal with this problem, many countries allow users to access the unused spectrum provided that they pay for the usage. Although accessing unused spectrum has been studied extensively, the proposed solutions do not consider the high mobility of the nodes. The connectivity of the proposed vehicular networks is another challenging problem for designing ITS.

Assume that vehicle A wants to send data to vehicle B and there is no vehicle on the path between $A$ and $B$. As a result, the data will not reach vehicle $B$. Hence, connection enhancement plays a vital role in designing ITSs. Another challenge is selecting the node for broadcasting emergency messages over the network. Bridge nodes should be identified when the network is built. Latency is another challenge for using short range wireless networks (SRWN) for traffic management. It is determined by the distance between the mobile vehicles, available spectrum, and the processing time for data traffic. Packet drops increase latency significantly [6].

These problems can be alleviated by using Wireless Mesh Networks (WMNs) which are proposed to extend networking services in personal, local, campus, and metropolitan areas. They provide cost effective communication, the same network capacity, reliability and performance as the wired network with the added advantages of flexibility and mobility for the users [5]. 
Different from the existing works, the owners in our study can hire their own spectrum to drivers. The hired spectrum is used to build SRWN for monitoring traffic and enabling drivers to exchange warning messages and perhaps other types of messages, and thus enhancing road safety and the driving experience. Our objectives include facilitating real time information about road conditions, thus minimizing crash probability among vehicles, utilizing unused spectrum, and enabling spectrum owners to generate extra revenue. Our analysis confirms the capability of our approach to enhance road safety under different road conditions. The major contributions of this paper are summarized as follows.

- A new spectrum-hiring scheme is proposed to improve the utilisation of the unused spectrum and analyze the economic aspects that arise due to the interactions between spectrum owners and drivers.

- A cost-effective wireless mesh technology is utilized to build a short-range wireless network for monitoring traffic and offer an efficient and reliable connectivity that enables drivers to exchange warning messages in order to minimize crash probability and enable spectrum owners to generate extra revenue.

- A new intelligent traffic management control system has been designed utilizing the unused spectrum where different levels of congestion, jointly considering spectrum prices, and the spatial distribution and performance of the SRWN have been analyzed.

The rest of the paper is organized as follows: Section 2 briefly surveys the relevant work; our assumptions and work environment are shown in Section 3; Section 4 describes the architecture of SRWN and the signaling protocol. Section 5 describes our scheme for road traffic control. The performance result of our scheme is evaluated in Section 6. Finally, the paper is concluded and future research directions are given.

\section{RELATED WORK}

Recent advances in wireless communications technologies have created many opportunities for the deployment of various ITS services in vehicular networks. In [9], the authors proposed CoVanet, a cognitive vehicular ad hoc network architecture that offers vehicles opportunistic access to unlicensed spectrum. In CoVanet, network topology and the available spectrum change over time due to high node mobility. The authors proposed a new scheme for short range communication among vehicles in [10]. It integrates clustering with contention-free-based medium access control (MAC) protocols. The elected cluster-head vehicle acts as the coordinator to collect/deliver real-time safety messages within its own cluster and forward the consolidated safety messages to the neighboring cluster heads. In [11], the authors considered the problem of accessing unused spectrum to provide quality of service (QoS) for data transmission in cognitive vehicular networks. Vehicles are able to reserve a dedicated channel for data transmission.

In [12], the authors presented a novel cognitive network architecture to assign the control channel dynamically. A control channel is used by vehicles to transmit safety-related information. Furthermore, a new cooperative spectrum sensing scheme is used to detect unused channels on the $5.8 \mathrm{GHz}$ ISM band. Vehicles use free channels to forward the data to a fixed infrastructure known as Road Side Units. In [13], the authors analyzed the statistical properties of the connectivity of vehicular ad hoc networks (VANETs) with user mobility. They assumed that the vehicles travel along a multilane highway that allows vehicles to pass each other. The probability distribution of the node population size and the vehicles' location distribution is derived. The authors of [14] investigated cognitive radio network for vehicular network, named as CR-VASNET. Various challenges and issues were discussed. Furthermore, new scheme was proposed to save stationary nodes' power and enlarge the network lifetime based on an optimization technique for relay node selection.

In [15], the authors studied the continuous communication availability in VANET. Continuous communication availability time is derived as functions of mobility and traffic load. In [16], the authors proposed new security procedure to protect VANET from untrusted data that may cause network congestion. The proposed procedure prevents a malicious user to supply false warning messages in the VANET, resulting in accidents or false alarms. A new strategy for node cooperation based makeup was proposed in [17]. In this strategy, neighboring nodes rebroadcast packets from source nodes to increase the reliability in vehicular networks. Vehicles are clustered in a multi-hop based novel approach named VMaSC in [18]. This new architecture VMaSC-LTE integrates 3GPP/LTE networks with IEEE 802.11p based vehicular networks. Novel wireless-based vehicle-to-pedestrian (V2P) collision avoidance using energy-efficient methods was proposed in [19]. The work employed an adaptive multi-mode (AMM) approach built on situation-adaptive beaconing to reduce power consumption for smart phones that are used for disseminating warning messages in vehicular networks. The authors proposed a new warning message dissemination mechanism (FRWD) in [20]. In the proposed scheme, emergency messages are delivered to a specific area in time and they are kept alive for a while. Two types of forwarders relay vehicles and backup vehicles are selected by balancing the forward progress and packet reception probability. Vehicles periodically broadcast warning messages with a reasonable time interval.

A cooperative collision avoidance (BIG-CCA) framework was proposed in [21] for preventing collisions. A vehicular sensor network is used by BIG-CCA to prevent chain vehicle collisions, which are common road accidents that occur when vehicles make sudden stops.

In [22], a new broadcast protocol was proposed. It can provide a low message overhead with low packet loss probability. A fuzzy logic algorithm is used to choose the next hop relay nodes. Moreover, network coding is used to improve the packet dissemination ratio without increasing the message overhead. A new proactive Cooperative 
Neighbor Position and Verification (CNPV) protocol was presented in [23] for warning message dissemination. CNPV detects nodes advertising false locations and selects optimal forwarder nodes so as to mitigate the impact of adversary users.

A new probabilistic model was proposed in [24] to mitigate the effects of the broadcast storm problem. The authors proposed a distributive cross-layer scheme for the design of the control channel in vehicular network [25] with three levels of broadcast services that are critical to most potential vehicle-safety related applications. The new scheme includes preemptive priority in safety services, dynamic receiver-oriented packet repetitions for one-hop emergency warning message dissemination, a multi-frequency busy tone and mini-slot within the distributed inter-frame space (DIFS) in IEEE 802.11, and robust distancebased relay selection for multi-hop broadcast of emergency notification messages.

There are significant differences in our approach not only due to considering the price of the spectrum but also due to studying the impact of the network performance on crash probability among vehicles under different conditions. This research aims to analyze the economic aspects that arise due to the interactions between spectrum owners and drivers. Spectrum owners will be motivated to offer spectrum for drivers if they expect to make extra revenue. We describe in detail the operations of how licensed bands can be hired to drivers for enhancing road safety. Previous studies [9-25] assume the spectrum availability for VANET. Furthermore, they assume the usage of spectrum is free where drivers can access licensed bands without paying any charge to the owners. Although these approaches enhance road safety significantly, the concept is not likely to be accepted in the current spectrum market since the spectrum owner do not have any financial incentive from driver's usage of spectrum. The direct financial incentives will promote spectrum owners to share it. The hired spectrum is used to establish VANET. Our scheme enables vehicles to access more spatial and temporal spectrum opportunities owned by different systems by a hiring process. The spectrum owners are expected to make more revenue due to the dramatic increase in the number of vehicles as well as the high mobility of vehicles.

Obviously, connectivity is the main challenge of designing ITS and thus we address this challenge through using a wireless mesh network. Mesh connectivity significantly enhances network performance and reduces packet drop probability. We consider the VANET design that is robust against low density network. In our work, VANET is guaranteed to remain connected with minimum number of vehicles. We have proposed a pioneering architecture for collecting and exchanging data regarding traffic conditions through VANET with a wireless mesh routers backbone infrastructure. It is worth indicating that the VANET performance improves in sparse network where the level of density is low. The above description reveals that it is really a novel area to use spectrum trading to enhance human safety using VANETs. This paper proposes a new scheme for road traffic safety which integrates the economical use of the radio-frequency spectrum, traffic conditions dissemination system, and features of wireless networks into an ubiquitous technology.

\section{NETWORK OVERVIEW}

In this section, the network model considered in the proposed approach is presented. First, WMNs are considered. WMNs have been deployed worldwide as cost-effective ubiquitous broadband connectivity offering a wide range of services in a given geographical area [26-29]. Although several network architectures have been studied, the mesh is undoubtedly one of the most popular owing to its many attractive features including rapid rollout, low capital costs, low power consumption, self-configuration and organization, high ability to integrate with other networks such as the Internet, and easy installation. Furthermore, a WMN is capable of using the basic radio frequency to provide robust, flexible mobile broadband communications to different communities through the readily reachable multi-hop connection [26, 27].

A number of representative WMNs have been deployed to operate in many cities and rural areas worldwide [27], [28], [23].

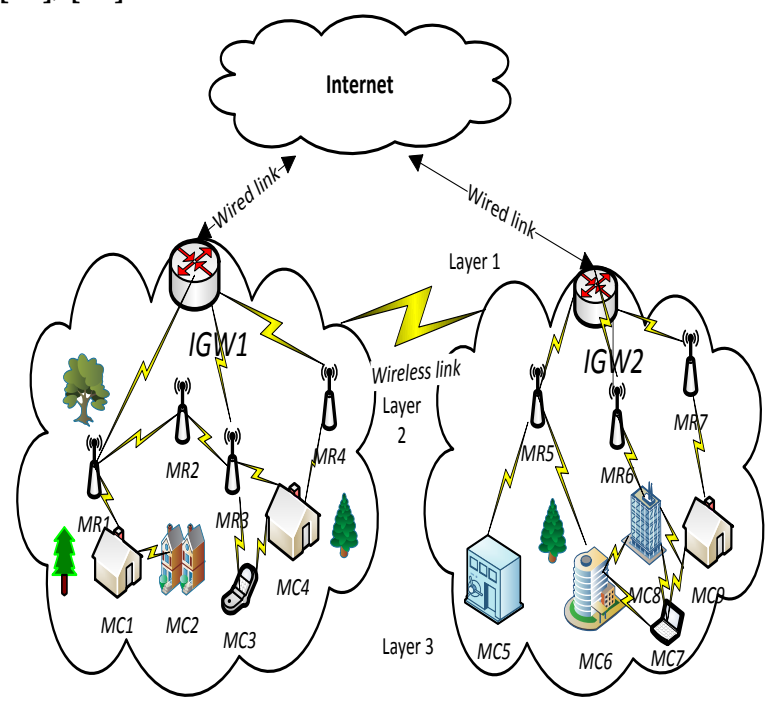

Fig. 1. The mesh architecture of wireless mesh

WMNs are multi-hop networks that are composed of wireless links that typically include static wireless mesh routers/gateways and potentially mobile clients, forming a combination of both fixed and mobile networks. Each client device is outfitted with radio communication gear that acts as a relay point for other nodes as well as a central orchestrating gateway. There are three layers in a typical WMN: Internet Gateway Layer (IGW Layer) that covers a domain, Mesh Router Layer (MR Layer) and Mesh Client Layer (MC Layer) as illustrated in Fig. 1. Gateways relay the message between the backbone Internet and nodes inside WMNs. Under each gateway, routers are connected to a backbone network via the gateway in the MR layer. The MR layer and MC layer include routing devices that use 
wireless radio and connect to the Internet backbone via two gateways IGW1 and IGW2 as illustrated in Fig. 1

WMN consists of three types of nodes: mesh clients (MCs), mesh routers (MRs) and Internet gateways (IGWs) forming the layers of the typical structure of WMNs as depicted in Fig. 1. To improve the flexibility of mesh networking, MR nodes are usually equipped with multiple wireless (radio) interfaces built on either the same or different wireless access technologies.

Each router and client is equipped with a single IEEE $802.11 \mathrm{~b}$ based transceiver. The spectrum is partitioned into non-overlapping channels ( 16 channels for each MR with 5 MHZ spacing with transmission and power mask restrictions similar to the ISM band), which is the basic unit of allocation. The network is divided into $M_{r}$ clusters (number of routers). Vehicles (MCs) are $M_{c}$ agile secondary nodes that act as mobile sensor nodes to sense the road traffic conditions. We assume vehicles are equipped with assistance of on-board sensors. These sensors include: GPS receiver, speedometer, accelerometer, and digital map to help a vehicle to specify road information.

Second, each driver willing to access the spectrum should pay the spectrum owner. The driver who pays for spectrum usage is referred to as the primary transmitter. Spectrum owner offers the available spectrum with prices for drivers. Vehicles are moving randomly and they change their speed arbitrarily. Vehicles select a direction randomly at each road intersection. Once the vehicle chooses a direction at an intersection, it moves straight until it arrives at the next intersection where it can change the direction.

Definition 1. WMN can be modeled as a network graph $G_{1}=$ $\left(V_{1}, E_{1}\right)$, where $V_{1}=\left(v_{1}, v_{2}, \ldots, v_{n}\right)$ is a set of $n$ nodes, including $M C s, M R s$ and IGWs and $E_{1}=\left\{(i, j): i, j \in V_{1}\right\}$ is the set of links (wireless and wired).

Definition 2. In our model, the graph $G$ is used for modeling the roads in the city. The graph $G_{2}=\left(V_{2}, E_{2}\right)$ consists of edge set $E_{2}$ and vertex set $V_{2}$. Edge $e_{i, j}$ represents the road over intersection $v_{i}$ and intersection $v_{j}$. The driver can drive from the intersection $v_{i}$ to the intersection $v_{j}$ directly. We refer to $e_{i, j}$ as a link, and a road is the undirected path connecting vertex $v_{i}$ with $v_{j}$.

Definition 3. In a given $G_{2}=\left(V_{2}, E_{2}\right)$, the number of $M R s$ for a particular edge $e_{i, j}$ can be calculated as follows.

$$
N_{m}=\left\lfloor\frac{\left|e_{i, j}\right|}{2 T}\right\rfloor+1
$$

where $T$ is the transmission range of $M R$. This configuration is illustrated in Fig. 2. Each edge $e_{i, j}$ is divided into $N_{m}$ cells and the position of mesh router $m$ in a cell $c_{i}$ is computed as follows:

$$
P\left(m, c_{i}\right)=2 T c_{i}-T
$$

Fig. 2. illustrates the architecture of the proposed network. We assume that each MR has a unique ID, which can be the MAC address of the node. MR constructs a traffic matrix $T_{m}$ which contains information about each vehicle in the road. This information includes: the speed of each vehicle on the road, and the position of each vehicle. This information is updated periodically through beacon messages. Our scheme can be divided into two tasks, namely cluster formation and density calculation.

\section{CLUSTER-BASED VEHICULAR SENSOR NETWORK}

Vehicles (i.e. MCs) in the network are divided into clusters. Each cluster has a coordinator (MR). In this clustered architecture, vehicles detect events in the road and then communicate the collected traffic information to a coordinator, which merges and analyzes the data. MRs have fixed locations but MCs are moving and changing their places arbitrarily.

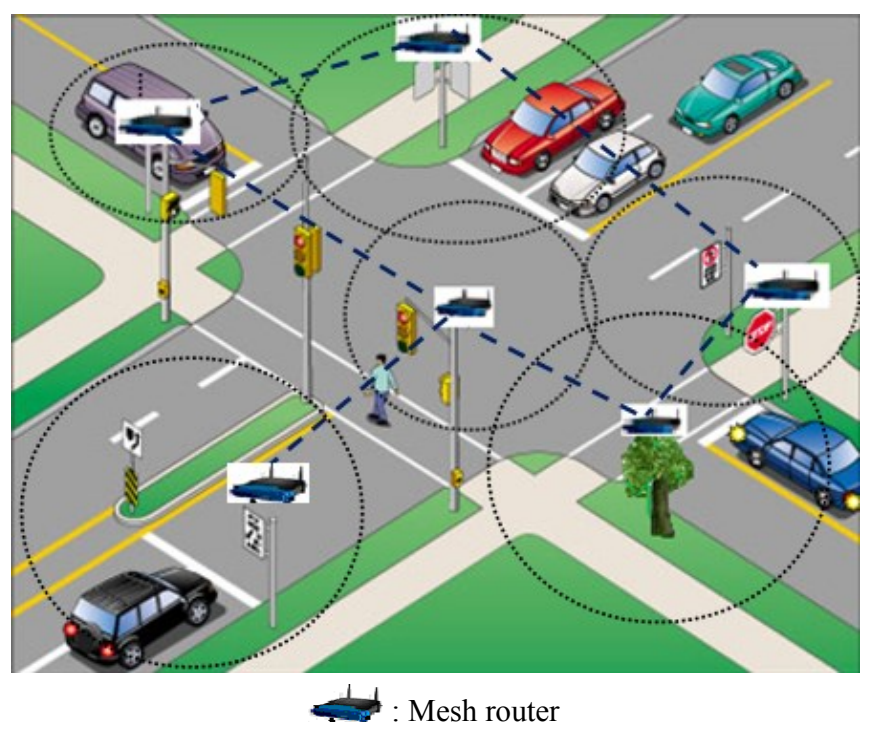

Fig. 2. Architecture of vehicular sensor network

Each MR has several MCs served by the MRs. Each MC in the network needs to obtain information about the routers in the network. This is done through router discovery. For clear exposition, the primary notations throughout the problem description are summarized in Table I. Each MC executes a distributed algorithm to select its cluster head (MR). Our clustering algorithm is initiated by simultaneously considering all MRs in the road and in the transmission range of MC as potential cluster heads. Information is exchanged among MCs and MRs iteratively until a subset of clusters emerges. Two kinds of beacon message are exchanged between MCs and MRs during forming clusters: invitations and acceptances. The invitation message is sent from the $j^{\text {th }} \mathrm{MR}$ to all MCs in its transmission range. The message contains the MR's ID and the price $r$ of accessing the channel. Each MC reflects the accumulated evidence for how well-suited the $j^{\text {th }}$ MR is to serve as the cluster head for the MC taking into account other potential MRs. Each $\mathrm{MC}$ sends an acceptance message to the MR reflecting the accumulated evidence for how appropriate it would be for 
the MR to be selected to serve the MC. Since each MC is moving and changing its place arbitrarily, the invitation and acceptance among the $i^{\text {th }} \mathrm{MC}$ and the $j^{\text {th }} \mathrm{MR}$ are updated at each iteration.

TABLE I

LIST OF RELEVANT NOTATION

\begin{tabular}{|c|c|}
\hline Notations & Description \\
\hline$G(E, V)$ & The underling road network \\
\hline $\mathrm{MC}$ & Mesh client \\
\hline$e_{i, j}$ & A network edge \\
\hline MR & Mesh router \\
\hline$N_{m}$ & The number of MRs for a particular edge $e_{i, j}$ \\
\hline$T$ & The transmission range of $M R$ \\
\hline$P(m, c)$ & The position of mesh routermin cellc \\
\hline$S(j, i)$ & The power of $j^{\text {th }}$ MR's signal at $i^{\text {th }} \mathrm{MC}$ \\
\hline$t_{p}$ & The transmitted power \\
\hline$L$ & The path loss at distance $d$ \\
\hline 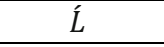 & The average path loss at distance $d_{0}$ \\
\hline$x_{\sigma}$ & $\begin{array}{l}\text { Zero-mean Gaussian distributed random } \\
\text { variable with standard deviation } \sigma\end{array}$ \\
\hline$P_{j}$ & The probability of a node to join a cluster \\
\hline$C$ & The spectrum cost \\
\hline$L_{i} W_{i}$ & $\begin{array}{l}\text { The length and width of a vehicle, respec- } \\
\text { tively }\end{array}$ \\
\hline$A_{i, j}$ & The area of the link $e_{i, j}$ \\
\hline$P_{v}^{i}$ & The space occupied by a vehicle \\
\hline$D_{j}^{i}(t)$ & $\begin{array}{l}\text { The traffic density at link } e_{i, j} \text { as the percent- } \\
\text { age of occupied space by vehicles at time } t\end{array}$ \\
\hline$p\left(h_{d}\right)$ & The probability of high demand state \\
\hline$O_{x}$ & The state of region $x$ (Occupied) \\
\hline$U_{x}$ & The state of region $x$ (unoccupied) \\
\hline$S_{x}^{\prime}$ & New state of region $x$ \\
\hline$a_{j}$ & Action that taken by a driver \\
\hline$C_{i}(S)$ & Cost function for each constraint \\
\hline$\gamma_{i}$ & The weight of $i^{\text {th }}$ constraint \\
\hline$J_{j}$ & $\begin{array}{l}\text { The system configuration action Jacobian } \\
\text { matrix }\end{array}$ \\
\hline
\end{tabular}

The MC selects its coordinator as follows:

$$
\max _{j \in M R_{r}} S(j, i) \text {, subject to } r_{i} \leq b_{i}, S(j, i) \geq \gamma
$$

where $S(j, i)$ is the power of the $j^{\text {th }} \mathrm{MR}$ 's signal at the $i^{\text {th }} \mathrm{MC}$ and $M R_{r}$ is the set of all MRs in the system. The first constraint states that the price of service $\left(r_{i}\right)$ that MC can pay for the $j^{\text {th }} \mathrm{MR}$ should be less than or equal his budget $\left(b_{i}\right)$. The MC fails to detect the MR's signal if the received power is less than $\gamma$. Thus, joining the $j^{\text {th }}$ cluster is a binary decision between the following two hypotheses:

$H_{1}: i^{\text {th }} \mathrm{MC}$ does not join $j^{\text {th }}$ cluster.

$H_{0}: i^{\text {th }} \mathrm{MC}$ joins $j^{\text {th }}$ cluster.

Signal strength at distance $d$ is computed as follows:

$$
S(j, i)=t_{p}-L
$$

where $t_{p}$ is the transmitted power and $L$ is the path loss at distance $d$, computed as follows:

$$
L=\bar{L}+10 n \log \left(\frac{d}{d_{0}}\right)+x_{\sigma}
$$

where $n$ is the path loss exponent, $d_{0}$ is the close-in reference distance, $\bar{L}$ is the average path loss at distance $d_{0}, x_{\sigma}$ is a zero-mean Gaussian distributed random variable with standard deviation $\sigma$. Standard deviation $\sigma$ describes the path loss model for an arbitrary location. Linear regression is used to estimate the value of $\sigma$. We assume the received power $S(j, i)$ has a Gaussian distribution. Let $P_{j}$ be the probability that the $i^{\text {th }} \mathrm{MC}$ will join the $j^{\text {th }} \mathrm{MR}$ cluster. It is computed as follows:

$$
P_{j}=p\left(H_{0} \mid j\right)
$$

Using Bayes theorem:

$$
P_{j}=\frac{p\left(J \mid H_{0}\right) p\left(H_{0}\right)}{p(J)}=p\left(J \mid H_{0}\right) p\left(H_{0}\right)
$$

We assume all MRs have the same chance of being selected by the $i^{\text {th }} \mathrm{MC}$. Hence, $p\left(H_{0}\right)$ is computed as follows:

$$
p\left(H_{0}\right)=\frac{1}{M_{r}}
$$

The $i^{\text {th }} \mathrm{MC}$ joins the $j^{\text {th }}$ cluster if the received signal is greater than a threshold $\gamma$ and the spectrum cost is less than $\mathrm{MC}^{\prime} \mathrm{s}$ budget. The probability of receiving a signal from the $j^{\text {th }} \mathrm{MR}$ with power greater than $\gamma$ is computed as follows:

$$
p(S(j, i) \geq \gamma)=\int_{\gamma}^{\infty} \frac{1}{\sigma \sqrt{2 \pi}} e^{\frac{(S-\pi)^{2}}{2 \sigma^{2}}} d s
$$

We assume that the prices from all the drivers are uniformly distributed. As a result, all outcomes have an equal chance of occurrence. The probability density function of pricing is given by [31]:

$$
f\left(r_{i}\right)=\left\{\begin{array}{cc}
\frac{1}{r_{i}-C} & , C<r_{i} \leq b_{i} \\
0 & , C>b_{i}
\end{array}\right\}
$$

where $C$ is the spectrum cost, $r_{i}$ indicates the maximum possible valuation of a spectrum band and $C$ is the minimum price that can be offered by the client. The main challenge in spectrum market is how to choose both the price and the offered size of spectrum. Demand uncertainty affects price and the offered size of spectrum. For low spectrum demand state, MCs offer less prices for getting services.

Definition 4. Assume that the $i^{\text {th }} M C$ offers a price $r_{i}$ to get service from the $j^{\text {th }} M R$. The request is accepted if and only if the 
price is greater than $C$ and the required bandwidth is less than the available spectrum.

The probability that the price of the spectrum is greater than the service cost and less than or equal to the budget of driver (i.e. $b_{i}$ ) is computed as follows:

$$
p\left(C<r_{i} \leq b_{i}\right)=\int_{C}^{b_{i}} f\left(r_{i}\right) d r_{i}
$$

By substituting (11) in (10) we get:

$$
p\left(C<r_{i} \leq b_{i}\right)=\frac{C-r_{i}}{b_{i}-r_{i}}
$$

The main challenge addressed below is how MR can choose both price and the offered spectrum size that meet all spectrum demands before knowing the state of the market (i.e. the demand for service).

Theorem 1: The optimal price $r^{*}$ which maximizes the MR revenue $V_{r}$ for a given spectrum size $K$ is computed as follows:

$$
K=Q\left(r^{*}\right)
$$

where $Q\left(r^{*}\right)$ is the spectrum demand in the high demand state.

The expected revenue is the additional revenue from selling an additional spectrum unit. For any new price $r$, the demand for spectrum either increases (i.e. high demand state) or decreases (i.e. low demandstate). Let $Q(r)$ represent the demand in high demand state after choosing price $r$, and $\Delta Q(r)$ be the amount by which demand is reduced in the low demand stat.

Proof: MRs' expected revenue is computed as follows:

$$
\begin{aligned}
E\left(V_{r}\right)= & p\left(Q\left(r^{*}\right)\right) r \min [Q(r), K]+(1- \\
& \left.p\left(Q\left(r^{*}\right)\right)\right) r \min [Q(r)-\Delta Q(r), K]-C K
\end{aligned}
$$

where $p\left(Q\left(r^{*}\right)\right)$ refers to the probability of high demand state. The deep insight from Equation (14) is that the optimal simple price rests on just two factors: marginal cost of offering spectrum unit (C) and the elasticity of demand (i.e. high demand state, and low demand state). The elasticity of demand is defined as a measure of the relationship between a change in the quantity demanded and a change in its price $(r)$. The marginal cost of spectrum is the additional cost of offering an extra spectrum unit. Under uncertain spectrum demand, the single first order condition captures how simultaneous changes in price and size of spectrum $(\mathrm{K})$ affect revenue. The first order condition with respect to the offered spectrum size $K$ has three regions that can be expressed as follows:

$$
\begin{aligned}
& \frac{\partial E\left(V_{r}\right)}{\partial K}=r-C, \quad K<Q(r)-\Delta Q(r) \\
& \frac{\partial E\left(V_{r}\right)}{\partial K}=p\left(Q\left(r^{*}\right)\right) r-C, \quad Q(r)-\Delta Q(r) \leq K<Q(r) \\
& \frac{\partial E\left(V_{r}\right)}{\partial K}=-C, \quad Q(r) \leq K
\end{aligned}
$$

In our solution, to select the optimal price, each partial derivative by itself represents the interaction between the price and the offered size of spectrum decisions. If, however, the demand is uncertain and the spectrum owner must choose the price and size of spectrum before observing the demand, the spectrum owner (MR) risks offering spectrum the owner can not hire (i.e. Equation (17)), a risk that the owner trades off with the risk of not being able to meet all demand (i.e. Equation (15)). Clearly, the revenue would be positive $\left(r^{*}>C\right)$ if $p\left(Q\left(r^{*}\right)\right)<1$. Otherwise, the expected revenues would be negative. The derivative in the first term is positive, thus $\mathrm{K} \geq Q\left(r^{*}\right)-\Delta Q\left(r^{*}\right)$. The derivative in the middle term is constant. If it is positive, then $K=Q\left(r^{*}\right)$, maximizes expected revenue. If it is negative, $K=Q\left(r^{*}\right)-\Delta Q\left(r^{*}\right)$. We apply Algorithm 1 for clusters construction.

\section{Algorithm 1:Cluster forming}

1: for $\boldsymbol{j} \leftarrow \mathbf{1}, \boldsymbol{M}_{\boldsymbol{r}}$ do

\section{2: $\quad$ Broadcast invitation message}

3: end for

4: for $\boldsymbol{i} \leftarrow \mathbf{1}, \boldsymbol{M}_{\boldsymbol{c}}$ do

5: $\boldsymbol{M R}_{\boldsymbol{r}}=\boldsymbol{M R}_{\boldsymbol{r}}$

6: $\quad$ for $\boldsymbol{j} \leftarrow \mathbf{1}, \boldsymbol{M}_{\boldsymbol{r}}$ do

7: $\quad \boldsymbol{S}(\boldsymbol{j}, \boldsymbol{i}) \leftarrow \boldsymbol{t}_{p}-\boldsymbol{L}$

8: $\quad$ end for

9: repeat

10: $\quad$ assign-head=false

11: $\quad$ candidate-head $\leftarrow \boldsymbol{m a x}_{\boldsymbol{j} \in \boldsymbol{M} \boldsymbol{R}_{\boldsymbol{r}}} \boldsymbol{S}(\boldsymbol{j}, \boldsymbol{i})$

12: $\quad$ if $\left(\boldsymbol{S}(\boldsymbol{j}, \boldsymbol{i}) \geq \boldsymbol{\gamma} \& \& \boldsymbol{r}_{\boldsymbol{i}} \leq \boldsymbol{b}_{\boldsymbol{i}}\right)$

13: $\quad$ cluster_head $(i) \leftarrow$ Candidate-head

14: $\quad$ assign-head=true

15: $\quad$ end if

16: $\quad$ else

17: $\quad \dot{M} \boldsymbol{R}_{\boldsymbol{r}}=\boldsymbol{M} \boldsymbol{R}_{\boldsymbol{r}}-\boldsymbol{j}$

18: until (Assign-head || $\mathbf{M}_{\boldsymbol{r}}==\emptyset$ )

19: end for 
Theorem 2: Each node (MR or MC) in the network sends exactly one message within two time steps.

Proof: In our algorithm, each MR sends an invitation message at $t_{0}$. After measuring the signal strength power of each received message, $\mathrm{MC}$ sends an acceptance message at $t_{1}$ to the MR with higher signal power.

Each MC should belong to only one MR. We proceed by induction hypothesis, showing that if $t=t_{n}$, then a node $j$ (MR or MC) sends either invitation or acceptance message. If $j \in M R_{r}$ then node $j$ sends an invitation message. If $j \notin$ $M R_{r}$, then node $j$ is an $\mathrm{MC}$ and it receives all the invitation messages at $t=t_{n-1}$ then it sends an acceptance message. Hence, by inductive hypothesis, at time $t_{n}$ nodes send messages. If all these messages are invitation messages $\left(M_{r}\right)$ then at time $t_{n+1}$ all messages are acceptance messages $\left(M_{c}\right)$.

Proposition1: The message complexity of the proposed clustering algorithm is $M_{r}+M_{c}$.

Proposition 2: Each MC in the network belongs exactly to one $M R$.

Proof: Each node $j$ sending an invitation message is an MR and it is the cluster head. On the other hand, if a node $j$ sends an acceptance message, then it belongs to the cluster whose MR has the highest signal power. Each node in the network is either MR (cluster head) or MC that is a vehicle node joins only one cluster head.

\section{Traffic Estimation Model}

The $i^{\text {th }} \mathrm{MC}$ reports its states and states of its neighbors periodically to the MR. Each report about the $i^{\text {th }} \mathrm{MCs}$ ' status is a vector $S_{v}^{i}$. The reported status vector at time $t$ by $i^{\text {th }}$ MC includes the following information: the space occupied by the vehicle $P_{v}^{i}$, position of the vehicle $\dot{\mathrm{p}}_{i}$, and instant velocity $v_{i}$. The space occupied by the vehicle $P_{v}^{i}$ is computed as follows:

$$
P_{v}^{i}=L_{i} W_{i}
$$

where $L_{i}$ is the length of vehicle and $W_{i}$ is its width. In our model, we define a traffic density at $\operatorname{link} e_{i, j}$ as the percentage of occupied space by vehicles at time $t$ which is computed as follows:

$$
D_{j}^{i}(t)=\frac{\sum_{k=1}^{N} P_{v}^{k}}{A_{i, j}}
$$

Here, $A_{i, j}$ is the area of the link $e_{i, j}$. Two vehicles collide with each other if they occupy the same cell at time $t$. Assume $O_{x}^{i}$ indicates the probability of occupying cell $x$ by the $i^{\text {th }} \mathrm{MC}$. Let $P\left(O_{x}^{i} \mid O_{x}^{j}\right)$ represent the likelihood of occupying cell $x$ by the $i^{\text {th }}$ vehicle given the same cell is occupied by the $j^{\text {th }}$ vehicle. $P\left(O_{x}^{i} \mid O_{x}^{j}\right)$ is computed as follows:

$$
P\left(O_{x}^{i} \mid O_{x}^{j}\right)=\frac{P\left(O_{x}^{i} \cap O_{x}^{j}\right)}{P\left(O_{x}^{j}\right)}
$$

In our work, the $j^{\text {th }}$ vehicle is able to detect the position of other vehicles at each link. Each detected position is indicated by the distance $\mathrm{d}_{i}$ from the $j^{\text {th }}$ vehicle where $i$ represents the $i^{\text {th }}$ vehicle. Generally, drivers' behavior can be represented by cost function that should be maximized or minimized (i.e. maximize the distance of the vehicles from the obstacles by reducing the speed). Let $S=\left[S^{1} \ldots S^{n}\right] \in$ $\mathcal{R}^{2 n}$ be the status of link $e_{i, j}$. Each $j^{\text {th }}$ action that is taken by a driver is represented by $a_{j}$. An action $a_{j}$ is related to the status of the link as follows:

$$
a_{j}=f(S)
$$

where $f(S)$ is a proper vector function. Taking a time derivative of (21) yields:

$$
a_{J}=\frac{\partial f(S)}{\partial a_{j}} \dot{S}=J_{j}(S) S \grave{S}
$$

where $J_{j}$ is the system configuration action Jacobian matrix. Each action changes the system configuration. These actions include:

- Decrease the velocity when traffic density approaches its critical value.

- Increase the velocity when the density level decreases significantly.

- Change the path in response to newly received information.

The following constraints should not be violated in the system:

- The diver should avoid driving onto occupied cells in the lane.

- The maximum speed is $110 \mathrm{~km} / \mathrm{h}$.

- The driver should not drive onto the pavement and should always stay in his/her lane and in the same gear.

Each constraint in our system can be represented by cost function $C_{i}(S)$, where $i=1 . \ldots n_{h}$, and $n_{h}$ represents the total number of constraints. The value of $C_{i}(S)$ increases when the $i^{\text {th }}$ constraint near to being violated. In order to minimize the cost function, the driver has to take an action that prevents constraint violation. An overall cost function, with $\gamma_{i}$ as the weight of the $i^{\text {th }}$ constraint, can be calculated as follows:

$$
T_{c}=\sum_{i=1}^{n_{h}} \gamma_{i} C_{i}(S)
$$


Definition 5.Suppose we have $N$ distinct vehicles at link $e_{i, j}$. Given that each link is divided into $M$ distinct cells, the total number of system states (i.e. number of ways of dividing the $N$ vehicles into $M$ cells) is computed as follows:

$$
f(N, M)=\left(\begin{array}{l}
N \\
M
\end{array}\right)
$$

Definition 6. Assume $O_{x}$ indicates that the cell $x$ is occupied and $U_{x}$ indicates cell $x$ is unoccupied and these states of cell $x$ represent a sensor reading of the $j^{\text {th }}$ vehicle. The two states of cell $x$ and new states $S_{x}$ are derived from the sensor of $j^{\text {th }}$ vehicle, using Bayes theorem:

$$
\frac{P\left(O_{x} \mid S_{x}^{\prime}\right)}{P\left(U_{x} \mid S_{x}^{\prime}\right)}=\frac{P\left(S_{x}^{\prime} \mid O_{x}\right) P\left(O_{x}\right)}{P\left(S_{x}^{\prime} \mid U_{x}\right) P\left(U_{x}\right)}
$$

In order to integrate other vehicle information $\hat{S}_{x}$ with the $j^{\text {th }}$ vehicle's measurements $\left(S_{x}\right)$,we assume $\hat{S}_{x}, S_{x}^{\prime}$ are independent of each other. Cell occupancy probability is computed as follows:

$$
\begin{aligned}
P\left(\widehat{S}_{x} \wedge S_{x} \mid O_{x}\right)=P\left(\widehat{S}_{x} \mid O_{x}\right) P\left(S_{x} \mid O_{x}\right) \\
P\left(\hat{S}_{x} \wedge S_{x}^{\prime} \mid U_{x}\right)=P\left(\widehat{S}_{x} \mid U_{x}\right) P\left(S_{x}^{\prime} \mid U_{x}\right)
\end{aligned}
$$

Using equation (20):

$$
\frac{P\left(O_{x} \mid \hat{S}_{x} \wedge S_{x}^{\prime}\right)}{P\left(U_{x} \mid \hat{S}_{x} \wedge S_{x}^{\prime}\right)}=\frac{P\left(O_{x} \mid \hat{S}_{x}\right) P\left(S_{x}^{\prime} \mid O_{x}\right)}{P\left(U_{x} \mid \hat{S}_{x}\right) P\left(S_{x}^{\prime} \mid U_{x}\right)}
$$

$\frac{P\left(O_{x} \mid \widehat{S}_{x}\right) P\left(S_{x} \mid O_{x}\right)}{P\left(U_{x} \mid \hat{S}_{x}\right) P\left(S_{x}^{\prime} \mid U_{x}\right)}=\frac{P\left(O_{x} \mid \widehat{S}_{x}\right) P\left(S_{x} \mid O_{x}\right) P\left(U_{x}\right)}{P\left(\bar{S}_{x} \mid \hat{S}_{x}\right) P\left(S_{x}^{\prime} \mid U_{x}\right) P\left(O_{x}\right)}$

In our model, we assume that the priori probability of a cell to be occupied by $j^{\text {th }}$ vehicle is 0.5 , (i.e. $p\left(O_{x}\right)=p\left(U_{x}\right)=0.5$ ), so that equation (24) can be written as follows:

$$
\frac{P\left(O_{x} \mid \hat{S}_{x}\right) P\left(S_{x} \mid O_{x}\right)}{P\left(U_{x} \mid \hat{S}_{x}\right) P\left(S_{x} \mid U_{x}\right)}=\frac{P\left(O_{x} \mid \hat{S}_{x}\right) P\left(S_{x}^{\prime} \mid O_{x}\right)}{P\left(U_{x} \mid \hat{S}_{x}\right) P\left(S_{x}^{\prime} \mid U_{x}\right)}
$$

Each MC sends its sensory report to the MR using a wireless data channel. In the processing phase, the MR solves data inconsistency and produces a final road status vector that contains the current status of the road. If the MR encounters any change in the road status, it disseminates the road status vector to all associated cluster members. The collected information about road status at MRs is used for managing the whole city. MRs exchange information periodically (based one one-hop transmission) to improve road safety. For example, a driver can use road information to avoid the regions where contention or congestion is high. Upon receiving a new status of road from MR, drivers check if there is any change. Road status vector can be used to resolve severe situations like traffic congestion and cope with emergency conditions.

Definition 7. For a target link $e_{i, j}$ the traffic condition of this link at time $t_{0}$ is defined as the percentage of occupied space at link $e_{i, j}$ by vehicles at $t_{0}$.

To compute the crash probability among vehicles, we use the model in [10]. The uncertainty on the future motion of vehicles is considered in [10] to compute the crash probability. A traffic matrix is generated using VanetMobiSim [30]. The road is divided into small cells and an accident has occurred if the center of two vehicles is in the same cell for all possible combinations of cells. The crash probability is measured as the percentage of overlap between the geometric shapes that represent the future motion of vehicles.

\section{PERFORMANCE EVALUATION}

In this section, we evaluate the performance of the proposed traffic management scheme. We consider an urban scenario of $15 \mathrm{~km} \times 15 \mathrm{~km}$, where vehicles are distributed randomly over the road, having speeds of $20-110 \mathrm{~km} / \mathrm{h}$. Roads are divided into a different number of cells. Assuming there is equal probability for selecting any direction at the intersection by the driver, the probabilities for each di-

\begin{tabular}{|c|c|c|}
\hline \multicolumn{2}{|l|}{ Parameter } & Value \\
\hline \multicolumn{2}{|c|}{ Number of MRs } & 100 \\
\hline \multicolumn{2}{|c|}{ Number of channels per MR } & 20 \\
\hline \multicolumn{2}{|c|}{ Number of messages per MC } & Random \\
\hline \multicolumn{2}{|c|}{ Type of interface per node } & $802.11 \mathrm{~b}$ \\
\hline \multicolumn{2}{|c|}{ MAC layer } & IEEE $802.11 \mathrm{~b}$ \\
\hline \multicolumn{2}{|c|}{ Transmission power } & 0.1 watt \\
\hline \multicolumn{2}{|c|}{ Packet size } & 512 \\
\hline \multicolumn{2}{|c|}{ Max Vehicle Speed } & $110 \mathrm{~km} / \mathrm{h}$ \\
\hline \multicolumn{2}{|c|}{ Warning message cycle } & $100 \mathrm{~ms}$ \\
\hline \multirow{4}{*}{$\begin{array}{l}\text { Simulation } \\
\text { Device }\end{array}$} & Intel i5 Core & $2.50 \mathrm{GHz}$ \\
\hline & Process cores & $2 \times 2.50 \mathrm{GHz}$ \\
\hline & RAM & $6 \mathrm{~GB}$ \\
\hline & OS & Windows 764 bit \\
\hline
\end{tabular}
rection are given by $P_{n}=P_{s}=P_{w}=P_{e}=0.25$. The parameters chosen for evaluating the algorithm and the methodology for the simulation are shown in Table II.

TABLE II

SIMULATION PARAMETERS 


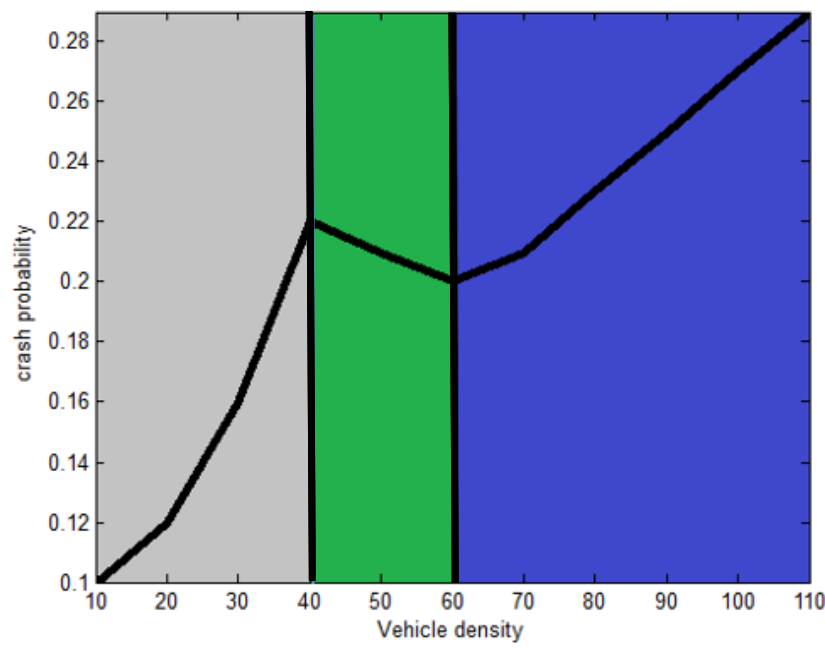

Fig. 3. Crash probability for different values of vehicle density

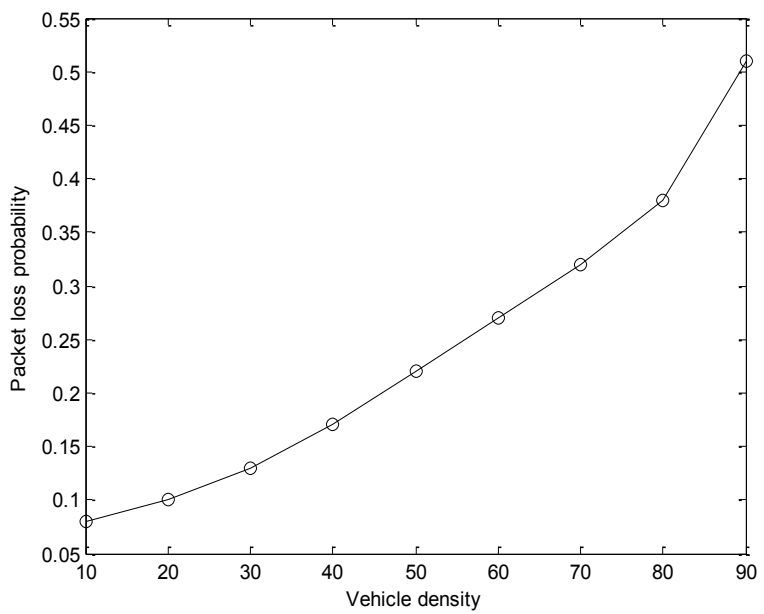

Fig. 4. Packet loss probability for different values of vehicle density

Fig. 3 shows the impact of vehicle density on crash probability. When the density is very small, the crash probability is small too. However, as the traffic flow increases and vehicle density approaches its critical value, drivers have to decelerate quickly to avoid crashing. Fig. 3 has three regions. In the first region (i.e. grey), the vehicle speed is low and traffic density is low. Clearly, fewer (serious) crashes have occurred in this region and the crash probability increases as the traffic starts to become congested, i.e. number of cars increases. For the second region (i.e. green), the connectivity of SRWN is improved significantly since the traffic density increases. In addition, we notice that the crash probability decreases despite the vehicles' speeds and traffic density are high. The crash likelihood decreases at this region due to exchanging warning messages between drivers. However, in the third region (i.e. blue), the crash probability starts increasing due to the lack of space and the degradation of SRWN performance. There is also another explanation of this behavior. In our work, the total number of channel resources is fixed in the network. By increasing the number of cars, the packet loss probability is decreased significantly, which improves the network performance in the second region of graph. However, after a certain number of cars, the packet drop probability grows due to congestion in the third region.

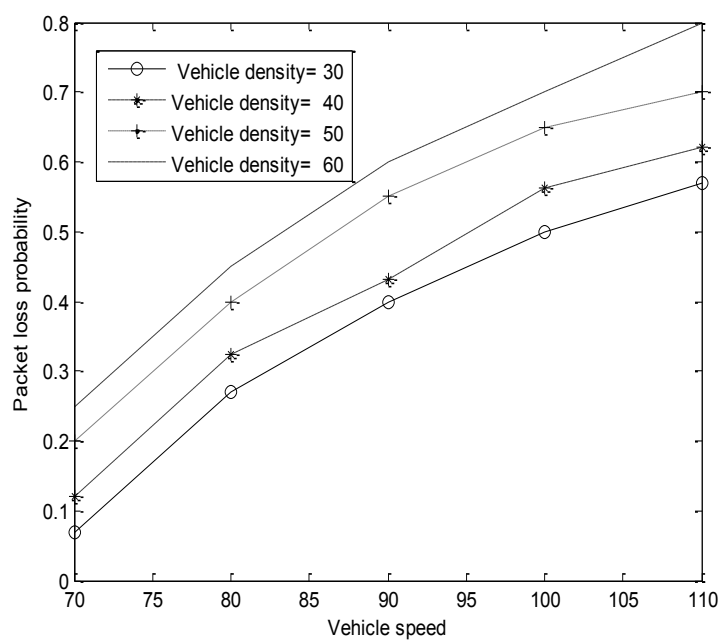

Fig. 5. Packet loss probability for different values of vehicle speed

Notice that, the packet loss occurs when packets travelling across a SRWN fail to reach their destination. It occurs basically due to network congestion. The occurrence of high density of cars within SRWN results in congestion, thereby causing significant performance degradation. For the higher values of density, a large number of cars compete for scarce SRWN bandwidth.

To study the effect of vehicle density on the performance of the SRWN, we measure the packet loss probability under various values of vehicle density. From Fig.4, we notice that as the density increases, the packet loss probability increases. From this experiment, we find that using wireless networks for road safety becomes less efficient in crowded road scenarios due to the network congestion. Mobility of vehicles is one of the most important factors that degrades the performance of wireless network and increases packet loss rates. Vehicle speed increases the crash probability significantly. Fig.5 displays packet loss probability under different values of vehicle speed. Fig. 6 shows the impact of vehicle speed on the bit error rate (BER) for different scenarios corresponding to an average vehicle speed of 70 $110 \mathrm{~km} / \mathrm{h}$. Clearly, the end-to-end BER increases when the vehicles are moving faster. In Fig. 7, we study the impact of the spectrum price on the performance of the SRWN under different scenarios of vehicles density. The price for the spectrum varies over time from $r=5$ (low price) to $r=30$ (high price). Fig.7 depicts that, as the price increases, the packet loss probability decreases due to the decrease in the traffic load. As the traffic load is increased, the packet loss probability increases significantly. In Fig. 8, we study the impact of the SRWN performance on the crash probability 
under different vehicles density. Fig. 8 depicts that as the packet loss probability increases (the performance of network degrades) the crash probability increases. This is because the chance of finding an appropriate channel for the warning message dissemination becomes very low. For higher packet loss probability, many warning messages are lost and the chance for avoiding vehicles' crash is decreased.

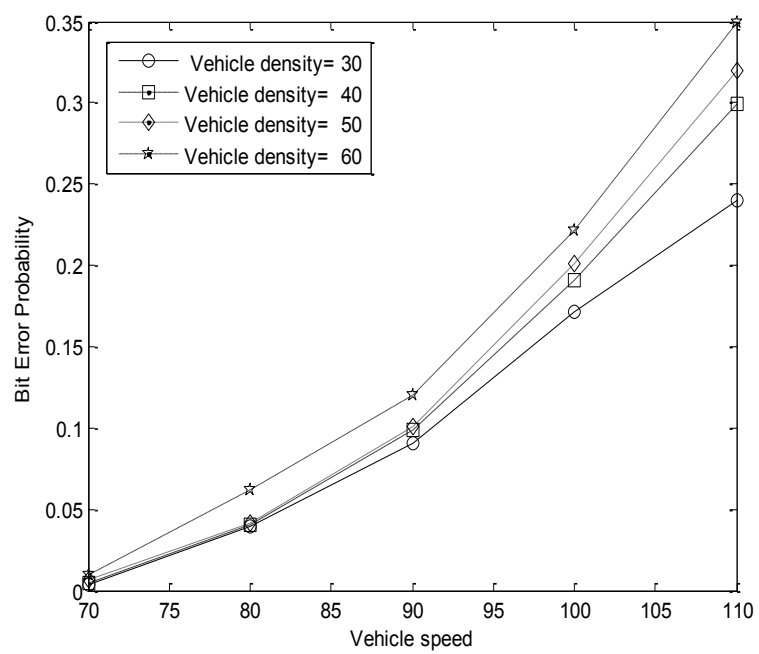

Fig. 6. Bit error rate probability for different values of vehicle speed

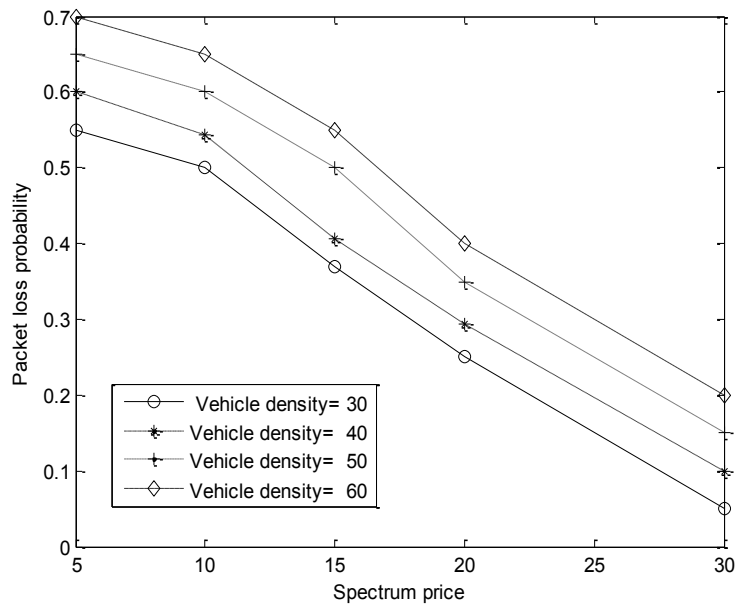

Fig. 7. Packet loss probability for different values of spectrum price

In Fig. 9, we study the response of drivers to unpredicted congestion that happens due to non-recurring highway incident or the presence of obstacle. Fig. 9 displays vehicle speeds of the first 15 vehicles in the road over time. It can be noticed that drivers slow down vehicle speed then they accelerate the speed after passing the obstacle. The first vehicle starts slowing down as the obstacle approaches, and other drivers follow the leader's speed upon receiving the warning message. The other drivers will experience the existence of congestion and they start deaccelerating their speed.

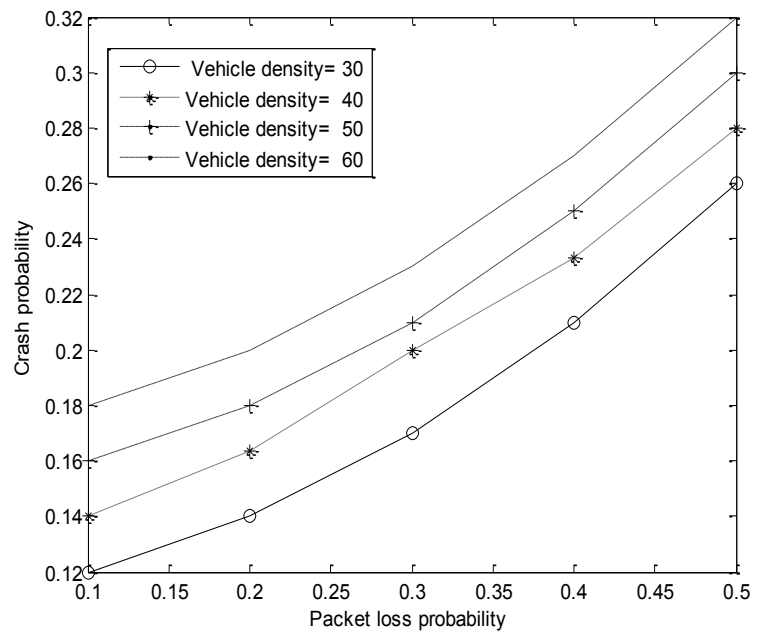

Fig. 8. Packet loss probability for different values of crash probability

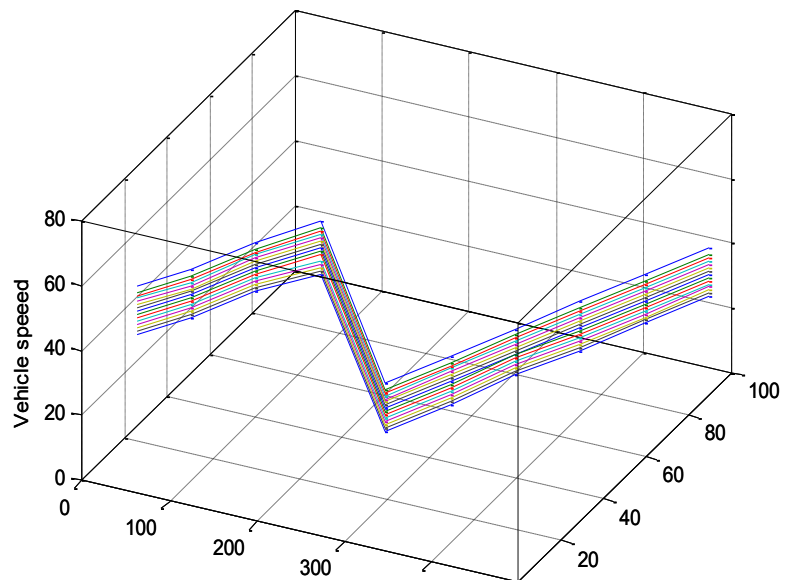

Fig. 9. Vehicles' speeds over time

\section{CONCLUSION}

In this paper, an intelligent traffic control system has been designed utilizing the unused spectrum. Different levels of congestion, jointly considering spectrum prices, and the spatial distribution and performance of the SRWN have been analyzed. A cost-effective wireless mesh community network technology has been deployed in our proposed cluster-based algorithm for managing spectrum over a road network. The aim is to minimize crash probability, utilizing unused spectrum, and enabling spectrum owners to generate extra revenue.

Simulation results have demonstrated that the proposed 
scheme can reduce crash probability among vehicles significantly, provided that the performance of the SRWN is not degraded. The research results can be applied for designing efficient traffic management systems. For future works, we will study the problem of identifying the routes with lower crash probability.

\section{REFERENCES}

[1] M. Ferman, D. Blumenfeld, and X. Dai, “An analytical evaluation of a real-time traffic information system using probe vehicles," Journal of Intelligent Transportation Systems, vol. 9, no. 1, pp. 23-34, 2005.

[2] S. Tao, V. Manolopoulos, S. Rodriguez and A. Rusu, "Real-Time Urban Traffic State Estimation with A-GPS Mobile Phones as Probes," Journal of Transportation Technologies, vol. 2, no. 1, pp. 22-31,2012.

[3] F. Yu and S. Biswas, "Self-configuring TDMA protocols for enhancing vehicle safety with DSRC based vehicle-tovehicle communications," IEEE J. Selected Areas in Communications, vol. 25, no. 8, pp. 1526-1537, 2007.

[4] H.A. Omar, W. Zhuang; L. Li, "VeMAC: A TDMA-Based MAC Protocol for Reliable Broadcast in VANETs," Mobile Computing, IEEE Transactions on, vol.12, no.9, pp.17241736,2013

[5] A. Alsarhan, A. Al-Khasawneh, A.Itradat, M.Bsoul,"Economic model for routing and spectrum management in cognitive wireless mesh network," International Journal of Networking and Virtual Organisations, vol. 12, no. 4, pp. 331-351, 2013.

[6] M. Di Felice, R. Doost-Mohammady, K. Chowdhury, and L. Bononi, "Smart radios for smart vehicles: Cognitive vehicular networks," IEEE Vehicular Technology Magazine, vol. 7, no. 2, pp. 26-33, 2012.

[7] X. L. Huang, J. Wu, W. Li, Z. Zhang, F. Zhu and M. Wu, "Historical Spectrum Sensing Data Mining for Cognitive Radio Enabled Vehicular Ad-Hoc Networks," IEEE Transactions on Dependable and Secure Computing, vol. 13, no. 1, pp. 59-70, 2016.

[8] Q. Yan, M. Li, F. Chen, T. Jiang, W. Lou, H. Y.T., C. Lu, "SpecMonitor: Toward Efficient Passive Traffic Monitoring for Cognitive Radio Networks," Wireless Communications, IEEE Transactions on, vol.13, no.10, pp.5893-5905, 2014.

[9] W. Kim, M. Gerla, S. Oh, K. Lee, and A. Kassler, "Coroute: a new cognitive any path vehicular routing protocol," Wiley Journal on Wireless Communications and Mobile Computing, vol. 11, no. 12, pp. 1588-1602, 2011.

[10] M. Althoff and A. Mergel, "Comparison of Markov Chain Abstraction and Monte Carlo Simulation for the Safety Assessment of Autonomous Cars," IEEE Transactions on Intelligent Transportation Systems, vol. 12, no. 4, pp. 1237-1247, 2011.

[11] D. Niyato, E. Hossain, and P. Wang, “Optimal channel access management with qos support for cognitive vehicular networks," IEEE Trans. on Mobile Computing, vol. 10, no. 5, pp. 573-591, 2011.

[12] A.J. Ghandour, K. Fawaz, H. Artail, M. Di Felice and L. Bononi, "Improving Vehicular Safety Message Delivery through the Implementation of a Cognitive Vehicular Network", Elsevier's Ad Hoc Networks Journal, vol.11, no. 8, pp. 2408-2422, 2013.

[13] M. Khabazian and M. K. M. Ali, “A Performance Modeling of Connectivity in Vehicular Ad Hoc Networks, "Vehicular Technology, IEEE Transactions on, vol.57, no.4, pp.2440-2450, 2008.

[14] M.Piran, Y. Cho, J. Yun, A. Ali, and D.Suh, "Cognitive Radio-Based Vehicular Ad Hoc and Sensor Networks," International Journal of Distributed Sensor Networks, 2014.

[15] M. Khabazian, M. Mehmet-Ali and S. Aissa, "Analysis of Continuous Communication Availability in Vehicular Ad Hoc Networks," IEEE Systems Journal, vol. 7, no. 1, pp. 137-150, 2013.

[16] M. A. Javed and E. B. Hamida, "On the Interrelation of Security, QoS, and Safety in Cooperative ITS," IEEE Transactions on Intelligent Transportation Systems, vol. PP, no.99, pp.1-15.

[17] S. Bharati and W. Zhuang, "CRB: Cooperative Relay Broadcasting for Safety Applications in Vehicular Networks," in IEEE Transactions on Vehicular Technology, vol. PP, no.99, pp.1-1, 2016.

[18] S. Ucar, S. C. Ergen and O. Ozkasap, “Multihop-ClusterBased IEEE 802.11p and LTE Hybrid Architecture for VANET Safety Message Dissemination," in IEEE Transactions on Vehicular Technology, vol. 65, no. 4, pp. 2621-2636, 2016.

[19] M. Bagheri, M. Siekkinen and J. K. Nurminen, "CloudBased Pedestrian Road-Safety with Situation-Adaptive Energy-Efficient Communication," in IEEE Intelligent Transportation Systems Magazine, vol. 8, no. 3, pp. 45-62, 2016.

[20] X. Zhu, C. Chen, Z. Pan and X. Guan, "Fast and reliable warning message dissemination mechanism in urban expressway VANETs,"2016 IEEE/CIC International Conference on Communications in China (ICCC), Chengdu, China, 2016, pp. 1-6.

[21] L. W. Chen and P. C. Chou, "BIG-CCA: Beacon-Less, Infrastructure-Less, and GPS-Less Cooperative Collision Avoidance Based on Vehicular Sensor Networks," in IEEE Transactions on Systems, Man, and Cybernetics: Systems, vol. 46, no. 11, pp. 1518-1528, 2016.

[22] C. Wu, S. Ohzahata, Y. Ji and T. Kato, “Joint Fuzzy Relays and Network-Coding-Based Forwarding for Multihop Broadcasting in VANETs," in IEEE Transactions on Intelligent Transportation Systems, vol. 16, no. 3, pp. 1415-1427, 2015.

[23] M. Fogueet al., “Securing Warning Message Dissemination in VANETs Using Cooperative Neighbor Position Verification," in IEEE Transactions on Vehicular Technology, vol. 64, no. 6, pp. 2538-2550, 2015.

[24] Y. Mylonas, M. Lestas, A. Pitsillides, P. Ioannou and V. Papadopoulou, "Speed Adaptive Probabilistic Flooding for Vehicular Ad Hoc Networks," IEEE Transactions on Vehicular Technology, vol. 64, no. 5, pp. 1973-1990, 2015.

[25] X. Ma, J. Zhang, X. Yin and K. S. Trivedi, "Design and Analysis of a Robust Broadcast Scheme for VANET 
Safety-Related Services," IEEE Transactions on Vehicular Technology, vol. 61, no. 1, pp. 46-61, 2012.

[26] A. Y. Al-Dubai, L. Zhao, A. Y. Zomaya and G. Min, "QoSAware Inter-Domain Multicast for Scalable Wireless Community Networks," in IEEE Transactions on Parallel and Distributed Systems, vol. 26, no. 11, pp. 3136-3148, Nov. 2015.

[27] S. Vural, D Wei and K. Moessner, "Survey of Experimental Evaluation Studies for Wireless Mesh Network Deploy-ment in Urban Areas Towards Ubiquitous Internet," IEEE Communications Surveys E Tutorials, vol. 15, no. 1, pp. 223-239, 2012.

[28] Weiyi Zhao and Jiang Xie, "DoMaIN, A Novel Dynamic Location Management Solution for Internet-Based Infrastructure Wireless Mesh Networks," IEEE Transactions on Parallel and Distributed Systems, vol.24, no.8, pp.1514-1524, Aug. 2013.

[29] Wei-Liang Shen, Chung-Shiuan Chen, Kate Ching-Ju Lin and Kien A. Hua, "Autonomous Mobile Mesh Networks,", IEEE Trans. Mobile Computing, vol.13, no.2, pp.364-376, Feb. 2014.

[30] VanetMobiSim,http://vanet.eurecom.fr

[31] Sengupta, S., Chatterjee, M., And Ganguly, S. An economic framework for spectrum allocation and service pricing with competitive wireless service providers. In Proc. of IEEE DySPAN (November 2007).

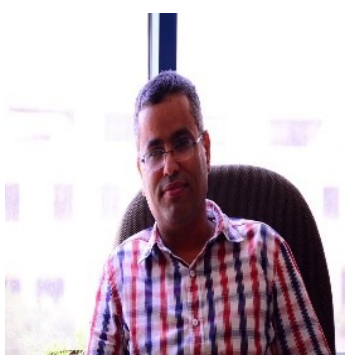

Ayoub Alsarhan received his Ph.D. degree in Electrical and Computer Engineering from Concordia University, Canada in 2011, his M.Sc. Degree in Computer Science from AlBayt University, Jordan in 2001, and B.E. degree in Computer Science from the Yarmouk University, Jordan in 1997. He is currently an Assistant Professor at the Computer Information System Department of the Hashemite University, Zarqa, Jordan. His research interests include Cognitive Networks, Parallel Processing, Cloud Computing, Machine Learning, and Real Time Multimedia Communication over Internet.

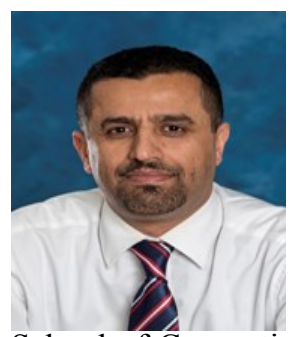

Ahmed Y Al-Dubai received his Ph.D. in Computing from University of Glasgow in 2004. In 2004, he joined the University of West London and then in 2005 he joined Edinburgh Napier University where he became a Professor and the Programm Leader of the Postgraduate Research Degrees of the School of Computing. He is the Head of the Networks Research Group. He has been working on the area of group communication algorithms, smart spaces and high-performance networks. He published in world leading journals including IEEE Transaction and in prestigious international conferences. He has been the recipient of several academic awards and recognitions and a member of several editorial boards of scholarly journals. He served as Guest Editor for over 20 special issues in scholarly journals and Chaired and
Co-chaired over 30 International conferences/workshops. Prof. Al-Dubai is a Fellow of the British Higher Academy and a Senior Member of the IEEE.

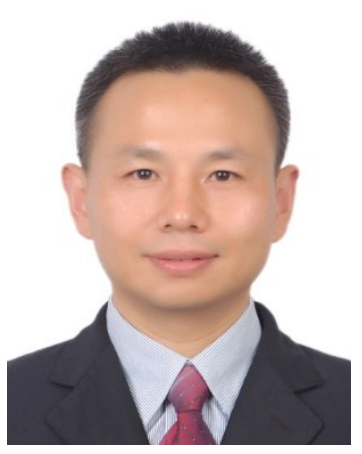

Gyong Min Professor Geyong Min is the Chair and Director of High Performance Computing and Networking (HPCN) Research Group at the University of Exeter, UK. He received the $\mathrm{PhD}$ degree in Computing Science from the University of Glasgow, UK, in 2003, and the B.Sc. degree in Computer Science from Huazhong University of Science and Technology, China, in 1995. He joined the University of Bradford as a Lecturer in 2002, became a Senior Lecturer in 2005 and a Reader in 2007, and was promoted to a Professor in Computer Science in 2012. His main research interests include Next-Generation Internet, Analytical Modelling, Cloud Computing, and Big Data.

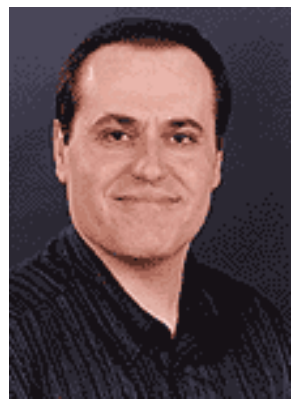

Albert Y. Zomaya is the Chair Professor of High Performance Computing \& Networking in the School of Information Technologies, University of Sydney, and he also serves as the Director of the Centre for Distributed and High Performance Computing. Professor Zomaya published more than 600 scientific papers and articles and is author, co-author or editor of more than 20 books. He is the Founding Editor in Chief of the IEEE Transactions on Sustainable Computing and serves as an associate editor for more than 20 leading journals. Professor Zomaya served as an Editor in Chief for the IEEE Transactions on Computers (2011-2014). Professor Zomaya is the recipient of the IEEE Technical Committee on Parallel Processing Outstanding Service Award (2011), the IEEE Technical Committee on Scalable Computing Medal for Excellence in Scalable Computing (2011), and the IEEE Computer Society Technical Achievement Award (2014). He is a Chartered Engineer, a Fellow of AAAS, IEEE, and IET. Professor Zomaya's research interests are in the areas of parallel and distributed computing and complex systems.

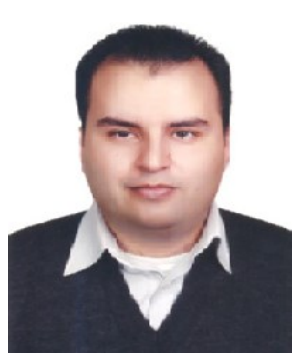

Mohammad Bsoul is an Associate Professor in the Computer Science Department of the Hashemite University. He received his B.Sc. in Computer Science from Jordan University of Science and Technology, Jordan, his Master from University of Western Sydney, Australia, and his Ph.D. from Loughborough University, UK. His research interests include wireless sensor networks, grid computing, cloud computing, distributed systems, and performance evaluation. 